EOMmun Communication et organisation

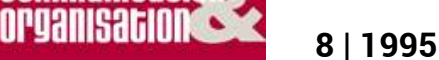

La communication au service de l'information?

\title{
Parcours obligés : du bon usage de la signalétique
}

\section{Dominique Pages}

\section{OpenEdition}

Journals

Édition électronique

URL : http://journals.openedition.org/communicationorganisation/1819

DOI : 10.4000/communicationorganisation. 1819

ISSN : 1775-3546

Éditeur

Presses universitaires de Bordeaux

Édition imprimée

Date de publication : 1 novembre 1995

ISSN : 1168-5549

\section{Référence électronique}

Dominique Pages, "Parcours obligés : du bon usage de la signalétique », Communication et organisation [En ligne], 8 | 1995, mis en ligne le 26 mars 2012, consulté le 01 mai 2019. URL : http:// journals.openedition.org/communicationorganisation/1819; DOI : 10.4000/ communicationorganisation.1819

Ce document a été généré automatiquement le 1 mai 2019.

(c) Presses universitaires de Bordeaux 


\title{
Parcours obligés : du bon usage de la signalétique
}

\author{
Dominique Pages
}

\begin{abstract}
L'espace de notre vie n'est ni continu, ni infini, ni homogène, ni isotrope. Mais sait-on précisément où il se brise, où il se courbe, où il se déconnecte et où il se rassemble ? (...) Nous cherchons rarement à en savoir davantage et le plus souvent, nous passons d'un endroit à l'autre, d'un espace à l'autre sans songer à mesurer, à prendre en charge, à prendre en compte ces laps d'espace.

(Georges Perec, Espèces d'espaces)
\end{abstract}

1 Dans un musée des beaux-arts de province, une femme attentive au marquage scrupuleux de la succession des salles s'étonne d'apprendre à la fin de sa visite l'absence du chef d'œuvre dont s'enorgueillit l'institution: la signalétique l'avait si bien intégré à ses repères directionnels que celle-ci ne l'avait pas dérangée. Dans un quartier central de Paris récemment réhabilité, un homme avance d'un pas pressé, ajuste nerveusement son regard et se fie si bien aux panneaux signalétiques qu'il ne voit pas cet autre homme, un habitué du quartier à l'évidence, qui fait un pas vers lui, prêt à lui indiquer le chemin, puis se détourne en haussant les épaules, laissant les signes remplir leur contrat.

2 L'air de rien, les systèmes signalétiques, qui se sont démultipliés depuis quelques décennies, ponctuent ainsi nos quotidiens, conditionnent nos déplacements et limitent nos errances au risque parfois de nous faire oublier les joies du hasard, les délices de l'égarement et l'intimité du regard. En forgeant ainsi notre sensibilité aux lieux et à l'environnement, ils modèlent, voire ritualisent, de plus en plus nos parcours au risque de créer chez chacun de nous une dépendance aux signaux, aux signes et aux symboles, parfois même au détriment de la signification et du sens des espaces traversés. Pourtant, malgré cette place croissante prise dans notre vie, aucune véritable réflexion ne semble avoir envisagé la nature, les fonctions et les limites d'un système signalétique. Pourquoi 
fait-on appel aujourd'hui à ce type de dispositif pour désigner et organiser les lieux, les espaces et les territoires contemporains ? Pourquoi leur conception mais aussi leur mise en œuvre et leur évaluation suscitent-elles tant de conflits? Quelle relation enfin la signalétique cherche-t-elle à instaurer entre son "commanditaire» (faute d'auteur effectif) et son destinaire? Au fil de définitions ouvertes et d'exemples centrés sur la mise en œuvre de dispositifs signalétiques par des villes et des musées, nous tenterons d'ébaucher ici cette réflexion.

\section{Signalisation et signalétique : de l'orientation au projet}

3 Comment définir de manière opératoire la signalétique et évaluer les liens ambigus qu'elle entretient avec la signalisation? Deux définitions proposées par la mission signalétique/images de la SNCF/RATP nous serviront de point de départ. Si elles montrent que toutes deux sont des systèmes d'orientation et d'information, elles permettent d'en dégager plus explicitement les différences tant formelles que fonctionnelles.

\section{De la convention technique à la proposition culturelle}

4 La signalisation y est ainsi définie comme "un système symbolique élémentaire de communication utilitaire (domaine du technique) basé sur l'apprentissage à la reconnaissance de signaux et de messages signes sur des bases formelles conventionnelles et sur l'engagement de la responsabilité bilatérale entre l'émetteur et le récepteur qu'entraîne ipso facto cette reconnaissance ». Sa lecture repose donc sur un code conventionnel, préétabli par une circulaire ou par un cahier des charges technique diffusés au niveau national. Son objectif premier est de permettre au conducteur des prévisions optimales dans le contrôle de son véhicule et au passant d'éviter l'accident. Son objectif second est de faciliter les parcours de l'automobiliste ou du passant d'un point à un autre, sans considération particulière pour les spécificités et les qualités des lieux croisés ni pour la cohérence des espaces traversés : elle répond donc à une logique de circulation, informant plus sur l'articulation des espaces et des territoires que sur leurs caractères propres ou leur singularité. Appelons donc signalisation des panneaux, fléchés ou non, susceptibles de s'organiser en un système normatif à des fins d'orientation et d'information et pouvant se déployer dans un espace ouvert, reliable à d'autres, dont la spécificité peut-être ignorée (voire occultée au risque d'une négation de la vie urbaine et d'une déréalisation de l'environnement ${ }^{1}$ ).

5 La signalétique, quant à elle, y est présentée comme "un système linguistique élémentaire de communication utilitaire (domaine culturel) basé sur l'expérience pratique et directe de la connaissance des messages et des signes messages sur des bases formelles déjà acquises et sur l'engagement de la responsabilité unilatérale de l'émetteur envers le récepteur (en ce qui concerne la non reconnaissance des messages) à charge pour l'émetteur d'améliorer indéfiniment l'ajustement de la forme au contenu de ce système de message tant sur le plan de la lisibilité que sur celui de la sémantique ». Appelons donc signalétique un système de signes, de symboles et plus largement de repères singuliers (linguistiques mais aussi symboliques) visant à valoriser ou à spécifier un lieu ou un espace dans une fin première de communication. Plus qu'un simple instrument d'information et d'orientation cherchant à rendre visible, elle est un vecteur 
d'image et de projet : en mettant en scène, d'une manière toujours orientée, les espaces qu'elle balise, elle répond à des fins non seulement symboliques et imaginaires mais aussi idéologiques. Son commanditaire ne cherche pas seulement à souligner par son biais la cohérence et l'unité du lieu, la singularité ou l'historicité de l'espace ou du territoire dont il a la responsabilité (économique, culturelle ou politique) mais à actualiser à travers elle un certain projet $^{2}$ : si la signalétique peut en effet combiner ses signes à ceux de la signalisation déjà présente, elle répond toujours à une intention stratégique et propose d'établir avec son destinataire, qu'il soit touriste, salarié, visiteur ou patient, une relation personnalisée voire un « contrat de lecture » toujours particulier qu'il faudrait analyser au cas par cas.

\section{Les courants d'air de l'histoire: des espaces clos aux espaces en mouvement}

D'emblée, les enjeux tant techniques, culturels que politiques de la signalisation et de la signalétique apparaissent à la fois complémentaires et divergents. S'inscrivant toutes deux dans la continuité des anciens systèmes de repérage et d'orientation que sont les bornes, les enseignes et les marques - corporatistes ou non - mais aussi les emblèmes, les sceaux et les armoiries (qui jadis ont permis au passant, au pèlerin ou au compagnon de se diriger dans des territoires plus ou moins hostiles), elles n'en coïncident pas moins à des mutations propres à la société contemporaine: signalisation et signalétique participent en effet du développement des logiques de flux et de mises en réseau des espaces et des territoires. Ces réseaux, qu'ils soient de transports, d'informations, de flux financiers ou d'idées, correspondent à des modes d'organisation coopératifs, adaptatifs et évolutifs impliquant de nouveaux rapports à l'espace et au temps, si caractéristiques de notre époque : en posant toute relation sociale sur le mode de la communication voire de la commutation, ils déstructurent les organisations spatiales traditionnelles. Entraînant une mobilité sociale croissante, ils peuvent provoquer chez les personnes un détachement grandissant par rapport aux lieux qu'ils habitent ou traversent et les amener à intégrer une certaine vision du monde selon laquelle l'espace et la matière comptent moins que la capacité à gérer des flux. Mais dans un même temps ils créent de nouvelles solidarités en maillant l'espace, tissent une toile d'araignée qui ne va pas sans ambiguïté. Ils rendent donc la pratique de l'espace contradictoire : d'un côté, ces réseaux permettent d'évoluer, de changer de lieu et de statut malgré la pesanteur du social et donc désenclavent; d'un autre côté, ils quadrillent, imposent leur propre métrique et donc entravent. Tout l'univers réticulaire ne prend sens que dans ce rapport antagoniste où le réseau, véritable Janus, reprend d'un côté ce qu'il accorde à l'autre ${ }^{3}$.

7 La signalétique et la signalisation actualisent ce paradoxe, le traduisent en signes : si toutes deux participent à faciliter les échanges et à rendre visible l'orchestration des espaces et des territoires par des repères fixes et adaptables, la signalisation tend à s'abstraire des territoires en privilégiant le chemin menant d'un point à un autre tandis que la signalétique semble plutôt découler d'une volonté de reterritorialisation, de symbolisation (voire de réenchantement) des lieux et des espaces. Certes la signalétique doit organiser les parcours mais dans un espace qu'elle circonscrit, qu'elle qualifie et parfois même « sur-identifie » de manière plus ou moins directive. 


\section{La crise de l'architecture comme système de représentation :}

8 Le développement des réseaux n'explique pas à lui seul l'émergence des systèmes signalétiques à côté des systèmes de signalisation : l'évolution de l'architecture l'éclaire plus concrètement. En s'hyperfonctionnalisant avec notamment le développement des CIAM (Congrès internationaux d'architecture) et en perdant ce caractère univoque qui lui permettait d'associer automatiquement forme et fonction, l'architecture contemporaine a rendu secondaires les points de différenciation des bâtis et des lieux jusqu'à ne plus savoir parfois suggérer par les formes qu'elle féconde une trame inévitable de cheminement. Espaces et lieux sont devenus de plus en plus insignifiants, impersonnels et substituables : l'hôpital, l'école et le centre administratif se sont mis à se ressembler; les liens traditionnels entre les formes urbaines et les relations sociales se sont déliés, rendant difficile toute élaboration d'un système de référence stable aux objets concrets ; l'effacement progressif des anciennes structures de proximité a non seulement généré l'inquiétude, le repli et la désaffection des espaces publics mais parfois motivé des actions de violences contre ces architectures anonymes: les banlieues mais aussi les écoles préfabriquées, les parkings et autres non-lieux en portent encore les traces.

9 Nombre de pratiques ont tenté de conjurer ce déficit de sens et cette difficulté de repérage: les méthodes de quadrillage et de découpage (ainsi les découpages institutionnels en quartiers, communes, département, régions) et les techniques de cartographie se sont démultipliées pour enserrer ces espaces indifférenciés ou, plus implicitement, pour en circonscrire les dangers réels et imaginaires. Une nouvelle volonté d'organisation spatiale s'est progressivement structurée : dans le sillage plus ou moins assumé de l'école de Chicago, l'urbanisme et la planification urbaine ont commencé dès les années 60 à délaisser le monumental au profit des parcours, du cheminement, de la rue et des espaces symboliques; la décentralisation, en cherchant à promouvoir de nouvelles formes de démocratie locale, a amplifié ce mouvement et resitué l'espace public au niveau du quotidien et de la proximité. La signalétique a été l'une des réponses à ces incertitudes et à ces inquiétudes : en aidant à circonscrire des espaces anonymes ou déqualifiés sans les enfermer, en les rendant plus intelligibles par une orchestration très cohérente des signes et en leur redonnant si ce n'est du sens du moins de la signification, elle s'est affirmée comme un outil privilégié de recomposition territoriale mais aussi comme un support d'identification suscitant des sentiments d'appartenance. Tout à la fois vectrice d'intégration et de déplacement, la signalétique s'énonce à la fois comme support d'appropriation des lieux et des espaces qu'elle organise mais aussi comme incitatrice à une plus grande autonomie de l'usager: grâce à ses indications, celui-ci se situe plus clairement dans l'environnement dont il a soudain une vision globale et synthétique et, dans un même temps, maintient une distance à l'autre auquel il ne fait plus systématiquement appel pour demander son chemin. En cela, la signalétique semble encourager un certain type de lien social et favoriser une socialité moins impliquante et moins frontale que celle des organisations traditionnelles.

\section{Des lieux, des espaces et des territoires à consommer.}

10 Si le développement de la signalétique constitue bien une réponse urbanistique à la crise de signification de l'architecture, il s'inscrit aussi dans l'extension des pratiques marketing à de nouveaux « produits ». Les logiques de communication institutionnelle se 
sont emparé de la signalétique pour valoriser, sans toujours les différencier, des entreprises et des villes, des magasins et des organismes publics, des musées et des centres hospitaliers, autant de lieux, de sites et de territoires qui sont devenus progressivement dans les années 80 des supports de consommation ou des médias de comportements à part entière. Envisagés comme des espaces relationnels devant favoriser les échanges et l'adhésion à un certain nombre de valeurs, ils se sont transformés en produits ou en marques à singulariser et à promouvoir. Mais, en inscrivant notamment les hauts lieux patrimoniaux et les espaces sociaux ou culturels dans un système de repérage sophistiqué, la signalétique ne les a-t'elle pas soumis à une réinterprétation appauvrissante et orientée ? En les organisant à partir d'une vision strictement communicationnelle, ne les réduit-elle pas à être des lieux et des espaces à consommer plus qu'à vivre? Difficile à ce point de notre réflexion de déterminer objectivement à quel moment il y a saturation d'information et déficit de sens pour le récepteur auquel s'adresse la signalétique. Les exemples qui suivent nous montrerons que cette réappropriation des lieux, des espaces et des territoires par le biais de la signalétique ne va pourtant pas équivoque.

\section{Signalétique des villes, signalétique des arts}

11 Ces exemples, centrés sur le musée et la ville, devraient permettre d'affiner et de nuancer ces définitions génériques. Dés les années 1970, les musées et les villes ont développé des systèmes signalétiques pour répondre à des objectifs d'information et de communication variés : la plupart de ces systèmes ont non seulement mobilisé de multiples acteurs, coûté cher mais aussi, le plus souvent, déçu leurs commanditaires.

\section{Les musées quadrillés : exaltation des œuvres ou célébration de l'espace muséal?}

12 En 1970 le musée du Vatican fut l'un des premiers à proposer à ses visiteurs une signalétique focalisée sur les temps de parcours (d'une heure et demie, de trois heures et de quatre heures) et ne découlant donc pas d'un projet véritable. Il faut attendre quelques années pour voir se dessiner en France des approches plus stratégiques qui susciteront souvent une forte réserve des conservateurs : le centre Beaubourg organise en 1976 un concours signalétique mettant en concurrence des bureaux d'architectes, des designers et des graphistes, suivi en 1985 par la Cité de la Villette, de 1983 à 1987 par le musée d'Orsay ${ }^{4}$ et en 1988 par le Musée du Louvre 5 . Ces initiatives s'inscrivent dans un contexte de remise en cause des politiques culturelles, dénoncées tant par les critiques que par les politiques pour leur élitisme et leur conservatisme : diffuser un "phénomène culturel », répondre aux attentes du visiteur et donc établir avec lui un lien d'une autre nature, s'intéresser aux modes de réception du patrimoine culturel deviennent les enjeux de la nouvelle muséographie. En déplaçant la question de la mise en espace des objets vers celle de leur mise en exposition et de leur rassemblement sous un étendard commun, la signalétique met en scène cette nouvelle conception de l'espace muséal: elle contribue ainsi non seulement à rendre l'objet intelligible, à guider les parcours du visiteur mais à convertir le musée en un média à part entière, vecteur de stratégies à facettes multiples.

Construite en 1982 sur les fondations des halles de la Villette, la Cité des sciences et de l'industrie fut créée pour diffuser une culture scientifique et technique en abordant les 
principaux thèmes scientifiques contemporains : l'espace, les énergies, l'informatique, les sons, les images, les volcans, la lumière mais aussi l'air et les matériaux. La composition même de la cité témoigne de cette vocation explicative et démonstrative et de cette volonté de multiplier les approches possibles de la vulgarisation scientifique : organisée en espaces autonomes (médiathèque, salle sciences actualité, cinéma, Centre de congrès, Explora, Eurocité, Cité des Enfants entre autres) construits par des équipes différentes, et divisée en 34 unités thématiques, la Cité cherche à se développer comme une ville avec ses quartiers et ses espaces libres, ses hauts lieux et ses détours. En 1985, les enjeux de la mise en œuvre d'une signalétique étaient donc à la fois simples et complexes : conçue en même temps que les expositions permanentes, celle-ci devait contribuer à la création d'une logique discursive dans un espace en construction sans pouvoir influer sur son organisation; elle devait lier les différentes unités entre elles pour faciliter tant l'orientation physique que la démarche de connaissance du visiteur qu'il soit français ou japonais, âgé de 7 ou de 77 ans; elle devait attribuer des noms à des espaces discontinus en évitant un vocabulaire trop technique et en privilégiant le ton ludique. Le cabinet hollandais retenu pour la signalétique interne à la Cité des sciences et de l'Industrie ${ }^{6}$, Total Design, proposa un projet privilégiant l'humour, les métaphores et la dimension pédagogique : les signes étaient simples et explicites, d'un graphisme libre et ludique, ne nécessitant pas pour être compris de document relais (plan, brochure, etc.). Ce fut pourtant un échec : d'un point de vue économique (le budget s'éleva au bout du compte à 15 millions de francs) mais surtout d'un point de vue d'organisation d'ensemble. L'évaluation montra en effet que les signes ne parvenaient pas à s'articuler en un système signalétique optimal: réalisé sans concertation véritable avec les concepteurs d'exposition ni avec ceux des autres supports de communication. Le système Total Design privilégia l'espace plus que les thèmes explorés, donnant des repères sur les allées centrales mais n'assurant pas le lien entre les expositions permanentes qui pourtant communiquent entre elles. Au moment de l'étude en réception, ce système devait se résumer en une accumulation de signes cherchant plus à diriger d'un point à un autre qu'à proposer un véritable parcours éducatif interactif. Pour combler ce déficit, la Cité recruta un responsable de la signalétique chargé de pallier aux dysfonctionnements du système : les signes s'accumulent pourtant encore aujourd'hui plus qu'ils ne s'organisent.

14 Avant 1988, l'espace immense et extraordinairement complexe que constituait déjà le musée du Louvre n'offrait guère à ses visiteurs d'itinéraire logique susceptible de faire découvrir sereinement ses multiples départements et la diversité de ses univers culturels : en 1988 fut donc commandé au cabinet de graphisme Widmer un système de repérage pour valoriser sans l'appauvrir l'éclectisme de ses collections et actualiser une nouvelle conception du visiteur mais ce système d'orientation ne survécut pas aux premiers travaux de rénovation du musée. Une nouvelle signalétique commandée à un cabinet de design américain, Carbone Smolan Associates, devait donc s'intégrer au processus de restructuration du musée mis en branle par l'Établissement public du Grand Louvre, entité juridique spécialement créée pour suivre ce chantier. Le système mis en œuvre coïncida avec l'innovation de la pyramide qui allait devenir la plaque tournante de cet immense complexe muséal. Il devait répondre à des objectifs et à des contraintes précis : respecter le caractère historique du lieu (palais et collections) mais en montrer la modernité (en pointant notamment le caractère innovateur de la pyramide, des boutiques, des politiques de programmation); faire preuve d'efficacité mais aussi de discrétion en évitant notamment un langage trop technique et trop logique; ne pas imposer un parcours obligatoire aux visiteurs mais leur laisser la liberté de choisir leur 
cheminement dans le musée; s'adapter au caractère éphémère des collections et à l'évolution des aménagements du musée. Le cabinet retenu répondit à ce cahier des charges par un projet inédit, fondé sur une organisation de type urbaine et sur une stratégie de communication soi-disant interactive : en divisant le palais en trois régions (Sully, Denon et Richelieu), elles-mêmes découpées en étages, eux-mêmes divisés en 10 arrondissements égaux en surface mais organisés de manière très disparate pour représenter les sept départements du musée, le système mis en place affirmait une logique centrée sur l'espace muséal ${ }^{7}$; en laissant au visiteur la possibilité de construire sa propre logique de lecture du musée, il privilégiait une approche " permissive » sur une approche strictement pédagogique; enfin en basant les parcours sur la lecture de nombreux repères spatiaux ludiques (fléchage singulier des chef-d'œuvre, plans divers fonctionnant sur des jeux de couleurs et des codes verbaux particuliers et indispensables pour diriger sa visite), il tendait à assimiler la visite à un jeu de piste, voire à une course au trésor. Les limites du projet furent rapidement mises en évidence par les conservateurs par le biais d'une étude d'impact: pour "jouer» au visiteur, il fallait impérativement entrer dans le code; la signalétique, jugée élitiste et complexe, demandait un effort de décryptage trop important et ne provoquait pas de conduites « réflexes » (je cite); en s'attachant plus à l'espace qu'aux collections, celle-ci s'abstrayait de son environnement et tendait à glorifier sa propre fonction. Pour combler ces déficits, le Louvre dut faire appel à une troisième agence, française, l'ADSA. Mais on peut se demander aujourd'hui, avec l'ouverture récente de l'aile Richelieu, la création simultanée du Carrousel du Louvre et l'ouverture en 1997 du "Projet Grand Louvre", sorte de mégacomplexe culturel regroupant entre autres le Musée du Louvre, l'Union centrale des Arts Décoratifs, l'Ecole du Louvre et les ateliers de restauration des musées de France, comment une équipe de signaléticiens parviendra à ordonner cette ville dans la ville, à proposer des itinéraires possibles sans transformer ce lieu en supermarché de la culture, comment elle réussira à donner une cohérence à un système de signes destinés à plus de 100 nationalités et communiquant sur des lieux aux vocations différentes.

Ces deux cas sont certainement exemplaires par l'ambition et la complexité des démarches mises en œuvre mais la plupart des musées n'ont à ce jour fait qu'ébaucher des projets signalétiques, c'est à dire dépassant un rôle d'information et d'orientation au profit d'une mission de communication à part entière. Pourtant, on peut dès maintenant comprendre les potentialités et les limites des signalétiques muséales. Non seulement celles-ci peuvent orienter et informer (tant sur les œuvres et les collections que sur les objets culturels et leur valeur d'usage) mais elles peuvent concrétiser des stratégies plus ou moins directives et sophistiquées: ainsi les stratégies "didactiques » des musées «mémoires» que constituent encore nombre de musées des Beaux Arts et d'Histoire, chercheront à axer l'information sur la conservation et l'accumulation des objets à partir d'énoncés dénotatifs au risque de transformer le musée en une succession d'instructifs passages obligés; les stratégies "pédagogiques » viseront à éduquer et utiliseront des artifices plus ou moins spectaculaires pour assurer l'échange entre l'émetteur (le musée et ses animateurs) et le visiteur; dans leur sillage, les stratégies « interactives» (surtout développées par les éco-musées, les musées des sciences ou à l'occasion d'expositions temporaires, il est vrai) viseront à faire participer ce visiteur et à ne pas cantonner les objets culturels dans une fonction univoque, faisant de la signalétique un support d'appel plus que d'orientation ou d'information. 
16 Malgré l'évidence de cette intention stratégique, la signalétique des musées n'a pas encore trouvé son statut ni acquis une légitimité claire. Tant sa difficile mise en cohérence des signes et des syntaxes qui la constituent que sa fréquente inadéquation à la mission officielle du musée - non pas faire circuler les visiteurs mais faire découvrir tant intellectuellement que sensiblement des œuvres et des pratiques culturelles manifestent sa faiblesse théorique et son indétermination déontologique. En quadrillant les salles, en rationalisant l'espace du musée, elle risque de marginaliser le singulier, de banaliser le rare, de lénifier l'esprit des collections. En ne parvenant pas véritablement à résoudre dès l'amont les évidentes controverses qui opposent les conservateurs aux signaléticiens, elle peut devenir l'objet d'un véritable et coûteux conflit culturel: spectaculaire, elle peut se résumer en un propos voire en une auto célébration du signaléticien dont la pratique est, rappelons le, plus proche de celle d'un urbaniste aménageur d'espace que de celle d'un conservateur; timide voire frileuse, elle abandonnera des signes disparates et déliés au visiteur égaré. En assimilant enfin le musée à un espace comme un autre - au mieux à une ville ${ }^{8}$ et au pire à une foire d'exposition-, elle tend à transformer les collections en de simples supports de communication voire de consommation que l'on peut «zapper» en toute bonne conscience puisqu'on ne fait que suivre les parcours prétablis par le musée. Depuis quelques années, les musées semblent avoir pris conscience de ces limites en développant des actions plus aptes à aider le public dans sa prise de connaissance des collections : programmation artistique et culturelle, visites et conférences, ateliers pédagogiques en sont les illustrations les plus actuelles.

\section{La ville balisée : du support d'image au projet de ville}

17 C'est de même dès les années soixante-dix que les villes se sont interrogées sur les moyens à privilégier pour valoriser leurs espaces et leur territoire mais aussi pour conjurer le rôle de relais et de connexion que la logique économique tendait à leur assigner. Parmi ces moyens, signalétique est apparue à même de faciliter les parcours de tout un chacun de manière conviviale et personnalisée tout en valorisant la politique (tant patrimoniale, urbanistique que sociale) du pouvoir local ${ }^{9}$. Mais l'audace, la maturité des démarches et l'investissement financier furent, selon les villes, d'intensités variables.

Les villes anciennes, à forte valeur patrimoniale, telles Bordeaux, Tours, Albi ou La Rochelle, ont mis en œuvre les projets signalétiques certainement les plus originaux, notamment à l'occasion du réaménagement de leurs centres anciens. La signalétique vise alors à personnaliser les itinéraires, à commémorer l'histoire des quartiers qui composent ces centres anciens dont elle met en valeur les lieux forts et les zones artisanales. Elle propose ainsi des parcours - quasiment des chemins de croix - où sont exaltés les ruines «édifiantes » et les lieux " pittoresques ${ }^{10}$ » et où sont désignés comme culturellement correct les bâtiments "classés » et les monuments "sauvegardés " (chapelles, églises, lieux historiques du pouvoir). La signalétique participe ici à une muséification de l'espace qu'elle ordonne: souvent recherchée dans son graphisme et dans l'adéquation de ses couleurs et de ses formes au caractère historique des lieux qu'elle désigne, elle constitue plus que jamais un discours sur la ville ou sur la partie de la ville qu'elle encadre, témoignant d'une politique culturelle, et plus précisément patrimoniale, quelque peu directive. De manière plus manifeste que les initiatives préalablement évoquées, les villes semblent se démarquer des logiques uniformisantes de type Decaux, tout en les tolérant. 
19 À l'opposé, Nanterre qui est sans doute l'exemple type de la démarche minimale: souffrant d'un tissu urbain sans véritable continuité et d'une intégration à une conurbation aux limites mouvantes, cette ville de banlieue emblématique a donné à sa signalétique une vocation essentiellement informative. Faute d'une identité et d'une territorialité claires, Nanterre a en effet mis en œuvre un système dont l'objectif premier est d'orienter et dont la portée utilitariste non seulement minimise la dimension esthétique et la visée communicationnelle mais tend aussi à réduire le projet signalétique à un simple système de signalisation. Celui-ci n'a fait jusqu'ici l'objet d'aucune réflexion stratégique, répondant plutôt à une politique du coup par coup et cherchant tant à maximaliser la lisibilité et la visibilité qu'à éviter une saturation du fléchage. Cette approche essentiellement techniciste est en partie due au fait que la signalétique dépend du département "services techniques" (au même titre que l'architecture d'ailleurs) et plus précisément du responsable « signalisation » dont l'objectif premier est de respecter l'instruction ministérielle de 1982 sur la signalisation directionnelle et d'assurer par la sobriété des panneaux et la discrétion de leurs emplacements, la sécurité des automobilistes : la seule pratique véritablement «signalétique » se résume à ce jour au récent fléchage installé pour conduire vers la Maison de la Musique, fléchage de type Decaux d'ailleurs, reprenant celui de la capitale. Il est donc singulier d'apprendre que malgré cette approche techniciste, la signalétique est évaluée à Nanterre par une multiplicité d'intervenants : le service technique bien sûr, la DDE, le service information et les élus mais sans concertation véritable pourtant avec le département communication.

Cergy Pontoise, "ville nouvelle» fruit d'une planification volontaire, a pour sa part élaboré une démarche signalétique complète, de sa conception à son évaluation, et ceci sous la tutelle de L'EPA qui a accompagné sa naissance ${ }^{11}$ ou plus précisément de son bureau d'études qui est composé de 'signaléticiens', d'urbanistes et d'ingénieurs, assistés de sociologues chargés de réfléchir aux mécanismes d'appropriation de l'espace par les administrés. Ce dispositif de signalétique s'est donné d'emblée plusieurs objectifs: prendre en charge les flux de circulation piétonniers et routiers; les différencier et les encadrer de manière directive et personnalisée; combler le déficit historique de cette ville sans mémoire; marquer le découpage en cinq quartiers et la cohérence de leur ensemble, envisagé comme un site urbain à part entière avec son unité géographique ; permettre à partir de cela l'affirmation d'une conscience d'agglomération; quadriller donc l'agglomération de façon homogène et conviviale, en donnant une idée claire et exhaustive de son offre de services et en insistant sur ses 'lieux-phares'(université, grandes écoles, conseil général, sentier de randonnée, etc.) - au risque évident d'un excès de citations. En mettant en place une étude de satisfaction des usagers dans l'intention de réajuster les modes de repérage déficients, la ville nouvelle a affirmé l'usage stratégique de la signalétique : non seulement celle-ci doit « conditionner » les parcours des habitants mais être l'occasion par son évaluation de mesurer les besoins d'orientation dans leur espace de vie des différentes catégories de la population. Mais, à force de concertation et peut-être aussi à cause d'un déficit de plus en plus criant d'harmonie architecturale de l'agglomération, Cergy est arrivé à un paradoxe : si la signalétique reste dépouillée dans son élaboration graphique, elle s'avoue bavarde et surabondante dans son système d'informations ${ }^{12}$.

21 Le dernier exemple urbain sera paradoxal mais riche en ouvertures. En 1992, la ville d'Ivry fit appel à l'agence Métaphores et Réalités pour installer une signalétique à l'emplacement d'une zone à l'état de friche: cette zone, alors désertée, située entre le 
port d'Ivry, la voie ferrée et le périphérique, devait accueillir dès 1993 le chantier de la TGB. Trois orientations furent données à ce singulier projet : élaborer un système de repérage et d'identification des lieux en le fondant notamment sur l'élément « eau » pour marquer l'emprise sur la ZAC de la proximité de la Seine et du passé portuaire du lieu; retrouver pour cela un vocabulaire de formes mêlant le portuaire, l'art et l'industrie. Ce projet fut ainsi actualisé par la création de balises de type fluvial et d'un système de repérage associant au thème de l'eau celui de la lumière et privilégiant le métal et la hauteur. La signalétique fit donc œuvre d'acte de fondation et se référa à l'efficacité symbolique selon laquelle la maîtrise des formes est censée procurer celle des choses : en énonçant ce qui allait être fait, elle articula le passé au futur et anticipa par le dire le faire 13 .

La signalétique urbaine se caractérise ainsi par une certaine hétérogénéité des pratiques : plus ou moins élaborées, les démarches intègrent de manière fluctuante la communication à sa conception et privilégient souvent les prestataires locaux (services techniques de préférence) à la fois pour des raisons de coût et de maintenance. Par ailleurs des initiatives audacieuses comme celles mises en œuvre dans les années 1980 par de grandes villes de province ou des banlieues en mal d'image semblent aujourd'hui s'essouffler au profit d'actions de communication plus participatives et moins spectaculaires mais aussi plus économiques et moins soumises à l'obsolescence : celle des signes trop marqués par la " modernité » et celle du projet peut-être trop lié au cycle des mandats. Mais une question reste ouverte : en se définissant comme un code d'interaction et un procédé de gestion des relations, la signalétique peut-elle être garante d'urbanité ?

\section{Quelle signalétique pour quelle appropriation de l'espace?}

Proposant certains types de parcours et en excluant d'autres, la signalétique témoigne d'une façon très contemporaine d'organiser l'espace. En ordonnant les espaces de notre quotidienneté selon les modèles propres de la culture dominante, elle en fait des « espaces symbolisés » au sens anthropologique du terme. Cette symbolisation qui vise à «rendre visible à tous ceux qui fréquentent le même espace un certain nombre de schèmes organisateurs, de repères idéologiques et intellectuels qui ordonnent le social ${ }^{14}$ " peut en effet prendre selon l'époque et le lieu, selon le groupe humain à qui elle se destine, des formes variables. Ainsi envisagée comme une métaorganisation de l'espace qui régente l'ordinaire, la signalétique apparaît bien être une mise en discours de l'institution ou mieux une production par l'institution d'un discours sur elle-même : en cela, elle est autoréférentielle et pourrait faire l'objet d'une étude approfondie de ses modalités d'énonciation (l'énonciation signalétique) et de ses effets, tant construits que réels. Etude au cas par cas qui permettrait de montrer en quoi la signalétique est bien un dispositif représentatif ${ }^{15}$ possédant deux dimensions - la première, transitive, représentant quelque chose, son objet, que ce soit le quartier, la ville, le musée; la seconde, intransitive ou réflexive, "se représentant représentant quelque chose ", son sujet (ici, l'autorité municipale ou muséographique) - et qui amènerait, en aval, le chercheur à évaluer les dissonances existant entre effets construits et effets réels. Une étude en réception permettrait en effet de savoir dans quelle mesure cette manipulation formelle modifie les réalités autochtones et participe à la maitrise des espaces et des territoires saisis dans le filet de ces représentations. Cette phase, centrée tant sur 
l'analyse des opinions et des attitudes individuelles et collectives que sur l'étude des ajustements de trajectoires et de l'adaptation des allures, mettrait en évidence tant les résistances des récepteurs à cette organisation visuelle que la complexité de la construction cet espace public (au sens figuré comme au sens propre) : le contrôle de l'espace public, envisagé comme lieu de régulation sociale, passe en effet par des règles, des conventions et une auto organisation autrement plus subtiles que le seul ordre visuel.

\section{Systèmes d'utilité publique ou simples dispositifs idéologiques ?}

généralisée des espaces qu'elle opère ne vont pas sans contradictions avec ses missions premières d'information et de communication: trop ostentatoire ou trop esthétisante, celle-ci peut ne pas coïncider avec la culture de ses destinataires et défavoriser toute possible identification; en surévaluant la qualité intrinsèque du lieu ou de l'espace qu'elle désigne, elle peut générer une relation d'incertitude voire un sentiment de perte de contact avec le réel, c'est-à-dire avec l'environnement immédiat. A force de porter un discours officiel et schématique sur les espaces, la signalétique peut aller jusqu'à créer un pseudoréel qui rend inutile la visite, superflu le détour. L'exemple, si bien évoqué par Marc Augé, de ces innombrables panneaux qui bordent les autoroutes et citent tous les hauts lieux des villes qu'on longe mais ne traverse plus est en cela édifiant : «Ce sont des textes disséminés sur le parcours qui disent le paysage et en explicitent les secrètes beautés. On ne traverse plus les villes mais les points remarquables sont signalés par des panneaux où s'inscrit un véritable commentaire. Le voyageur est en quelque sorte dispensé d'arrêt et même de regard(...). Il (le parcours autoroutier) est doublement remarquable: il évite, par nécessité fonctionnelle tous les hauts-lieux dont il nous rapproche; mais il les commente (...) en sorte que l'espace abstrait qu'ils (les conducteurs) sont conduits régulièrement à lire plus qu'à regarder leur devient à la longue étrangement familier ${ }^{16} »$.

Alors, faut-il éradiquer les systèmes signalétiques pour retrouver une urbanité plus «chaude " ou pour pouvoir encore se perdre dans le labyrinthe des musées? D'autres pistes, moins radicales et essentiellement urbaines, se dessinent ici et là : si les bornes interactives et l'apparition de signalétiques sonores, voire olfactives, ne font qu'amplifier l'imposition de «sens " qui caractérise la signalétique, l'évolution de l'art urbain et l'émergence de signalétiques marginales et spontanées, semblent en effet proposer des parcours plus libres, moins directifs et souvent plus éphémères. Ces pistes proposent des représentations symboliques moins stéréotypiques que celles précédemment envisagées et tentent d'appréhender les lieux et les espaces dans leur complexité, faite d'interactions multiples dans l'espace et dans le temps. 


\section{Echappées belles}

27 L'actuel renouvellement du mobilier urbain et de l'aménagement des musées permet ainsi d'espérer un assouplissement des pratiques signalétiques des années soixante-dix/80 : en s'intéressant plus aux qualités fonctionnelles qu'à la valeur esthétique et culturelle des dispositifs visuels, les designers semblent actuellement substituer aux pratiques de bel ordonnancement des pratiques plus subtiles et moins voyantes. Les plus connus d'entre eux, Foster, Wilmotte, Stark, Jourde et Perraudin, s'intéressent aujourd'hui plus aux lieux intermédiaires (pour la ville: places, trottoirs, voiries auxquels on accorde enfin officiellement le statut d'espaces publics) ou aux situations (pour les musées: scénographie et nouveaux supports interactifs) qu'à la lisibilité horizontale des rues et des salles. Ces interventions adaptées aux sites et à la topographie des espaces peuvent intégrer dans leur conception les usagers et semblent marquer un possible renouveau des «identités urbaines » et des politiques culturelles des musées. Leurs actions dépassent le formatage des espaces pour une gestion plus responsable à même de faire coincider espaces publics visuels et espace public de la discussion.

Dans un même temps, des signalétiques relevant d'initiatives privées s'ébauchent au fil des rues et des espaces abandonnés. Ainsi l'approche singulière de Nemo qui applique depuis des années dans l'Est de Paris (Belleville et ses environs ${ }^{17}$ ) ses pochoirs sur les murs et les façades d'immeubles promis à la disparition et propose aux riverains et aux flâneurs une sorte de signalétique intimiste: par ses exclamations et l'étrangeté du personnage qu'elle met en scène, celle-ci pointe et poétise à la fois les stigmates de la ville, ses zones murées en quête d'un incertain devenir, offrant au flâneur des itinéraires atypiques et initiateurs d'interrogations ; de même de jeunes « artistes » new-yorkais, à la demande de familles dont un proche a été assassiné en pleine rue dans les quartiers difficiles de la ville, ornent d'une fresque, riche en symboles et en inscriptions enchevêtrées, un mur proche du drame en l'honneur du défunt: tout comme les marquages de Nemo, ces singulières peintures funéraires, qui trouvent leur inspiration tant dans l'iconographie chrétienne traditionnelle que dans un métissage d'autres cultures, deviennent l'objet de parcours et d'ouvrages singuliers ${ }^{18}$.

Cette invitation à envisager d'autres systèmes de repérage et de valorisation visuelle s'achèvera par un retour vers le passé : un ouvrage consacré à " Paris par ses enseignes ${ }^{19}$ " datant du dix neuvième siècle vient d'être récemment réédité. Il renvoie aux enseignes d'antan, celles d'avant la numérotation et la nomination des rues. Ces enseignes qui affichaient en rébus leur raison sociale surprennent par leur iconoclasme : calembours et devinettes élaborées, jeux de mots et de couleurs témoignent d'une invention et d'une liberté insoupçonnées. Ne cherchant pas à imposer de logique d'ensemble, elles faisaient déborder sans précaution aucune l'espace privé sur l'espace public: une nostalgie s'installe quant on sait qu'aujourd'hui toute mise en place d'enseigne et de panneaux est soumise à autorisation préalable et est assujettie au paiement de droits de voirie. Belle leçon de choses urbaines. 


\section{L'évitement du temps et des temporalités urbaines : de l'idéologie à l'utopie?} le temps et les différentes temporalités qui interfèrent dans les espaces qu'elle organise : en mettant au même niveau la cathédrale du XII ${ }^{\mathrm{e}}$ siècle et la préfecture, ancienne demeure seigneuriale du XVII ${ }^{e}$, l'échoppe folklorique et le centre culturel à peine achevé, en rapprochant par une mise en signes homogènes (textuels et iconiques) le tableau de la Renaissance italienne et celui de l'expressionnisme allemand, la signalétique ne réduitelle pas ces lieux à une pure spatialité et ces œuvres à de simples objets référentiels d'une époque? N'entretient-elle pas ainsi une relation paradoxale avec la mémoire et l'histoire?

Certes, elle donne forme au temps en organisant les parcours mais elle n'incite pas au temps de la rencontre et de la confrontation, au temps de l'échange construit. En circonscrivant des espaces et en suggérant des déplacements, elle fixe une certaine normalité des comportements et, derrière une uniformité de surface, minimise l'hétérogénéité profonde des acteurs qui occupent cet espace. Stratégique mais peu politique, elle constitue, pour ce qui est de la ville, un support d'urbanité et un relais de citoyenneté limités; pas plus qu'elle n'incite à la participation et qu'elle n'envisage la ville comme une forme dans le temps (avant d'être une forme dans l'espace), elle n'ouvre véritablement à une réflexion sensible et informée sur la recomposition des espaces qui nous entoure et sur le processus dynamique de cette recomposition.

égiant une mémoire officielle, extérieure et matérielle sur des mémoires plus diffuses voire plus confuses, la signalétique ne témoigne en effet ni des enchaînements complexes ni du travail du temps : isolés de leur contexte historique que signifient cette chapelle romane, cette demeure huguenote qu'elle désigne? que signifie ce panneau coincé entre la rue du Nil et la place du Caire et qui vante les charmes sulfureux de la cour des miracles dont il ne reste pourtant aucune trace à l'entour? que les guerres napoléoniennes qui ont amené à rebaptiser les rues du quartier n'ont pas eu lieu, que le Sentier et son épopée textile n'ont jamais existé ? En proposant des arrêts sur image ou en sélectionnant des morceaux choisis dans un ensemble complexe, en figeant en des représentations idéalisées et univoques des lieux et des espaces pourtant évolutifs, la signalétique ne renvoie-t'elle pas en fait au processus utopique qui réfute la contradiction, dessine des espaces clos et met entre parenthèses le temps ? C'est sur cet évitement du temps, celui des mémoires composites, des histoires plurielles et des usages sociaux, qu'ont à s'interroger les signaléticiens, les designers de l'environnement et leurs commanditaires pour renouveler leurs approches formelles de l'espace. 
NOTES

1. Risque dénonce par Dominique Fleury dans un article consacré à la signalisation routière : »la gestion du trafic s'intéresse aux flux sur des segments linéaires et aux mouvements dans les points d'échange ; l'espace urbain est alors oublié au seul profit d'un fonctionnement optimisé du réseau. Cette démarche s'aggrave souvent d'un traitement très routier de l'espace public affecté à la circulation ", Rendre lisible la rue, in La Ville, CNRS, été 1994.

2. Le titre de la plaquette sur la signalétique éditée par le CETUR (Centre d'études des transports urbains devenu le CERTU, centre d'études sur les réseaux, les transports, l'urbanisme et les constructions publiques) est en cela édifiant: Marquer votre volonté var la politique de marquage.

3. Cette analyse est développée dans un recueil de textes consacré aux « Réseaux, territoires et organisation sociale ", sous la direction de Jean-Marc Offner in Problèmes politiques et sociaux, $\mathrm{n}$ - 740, décembre 1944

4. Beaubourg et la Villette ont retenu Jean Widmer pour prendre en charge leur image graphique (de la signalétique des lieux aux papiers à lettre).

5. Exemple cité par Sophie Razel, in Pour une définition de la signalétique dans l'espace muséal, mémoire de maîtrise d'information et de communication, Celsa Sorbonne, 1991.

6. L'agence Grapius ayant été retenue pour la Charte graphique d'ensemble.

7. Aux dépens de l'approche historique des conservateurs, centrée elle sur les collections et donc privilégiant les objets.

8. Une réflexion plus poussée sur cette ambition à devenir une ville voire une cité qui travaille sourdement les institutions muséales aujourd'hui reste à faire : quel projet citoyen s'y dessine implicitement?

9. La présentation de chacune de ces expériences se base tant sur des entretiens menés auprès des responsables de la mise en œuvre des systèmes signalétiques que sur les cahiers des charges et les études qui leur sont attenantes.

10. Les bornes mises récemment en place par la Mairie de Paris pour signaler au passant, par le biais d'un court récit gravé en lettres rouges sur fond brun, l'historicité du lieu ou du quartier qu'il croise ou traverse sont en cela exemplaires : "L'hôtel du Nord » au bord du Canal Saint Martin est ainsi pointé au même titre que "La Cour des Miracles" dans le Sentier. Ces brefs récits privilégient l'anecdote ou la spectacularité et restent fort réducteurs, n'apportant aucune clé de lecture pour la compréhension du lieu ou de l'espace désigné.

11. Espace public d'aménagement, établissement à caractère industriel et commercial qui a reçu du gouvernement mission d'aménager le vaste périmètre de l'agglomération de Cergy. Il mène pour le compte de l'Etat et des collectivités locales des études d'urbanisme et réalise les grands travaux d'infrastructures indispensables

12. Exemple cité par Valérie Salviac in La signalétique de la ville : une application inédite au service de la ré-appropriation de l'espace urbain, maîtrise d'information et de communication Celsa-Sorbonne, 1992.

13. Cette suprématie de la signalétique peut-être autrement illustrée par la tendance actuelle de l'architecture à s'intégrer elle-même à la signalétique comme emblème-phare de la ville à se faire support de communication à part entière: les commandes de monumentalité qui instrumentalisent ainsi l'architecture aux fins de légitimation d'un pouvoir politique nouvellement instauré mais aussi les produits immobiliers tendent à devenir, au même titre que la signalétique, une image de marque de la ville.

14. Marc Auge, Pour une Anthropologie des mondes contemporains, p.14, coll. Critiques, Aubier, 1994. 
15. Cette proposition s'inspire d'un article de Louis Marin, consacré à l'étude de cartes anciennes de la ville de Strasbourg: "La ville dans sa carte et son portrait ", in La représentation, éd. du Seuil, 1994.

16. Marc Augé, Lieux et non lieux, introduction à une anthropologie de la modernité, la Librairie du XX e siècle. Seuil, 1992.

17. Nemo a résolument choisi les zones dévastées puisqu'il déclare vouloir maintenant bomber Bogota, Beyrouth ou Bucarest (Libération, mars 1995).

18. Libération du 20.02.95 qui renvoie notamment à l'ouvrage de Martha Cooper et Joseph Sciorra, Bombages in Memoriam à New York, édition Thamson and Hudson, 1994.

19. Paris par ses enseignes, par le polygraphe Édouard Fourier, éd. La Bibliothèque. Cet ouvrage rappelle que certaines de ces enseignes étaient de vrais tableaux, peints par Chardin, Watteau, Greuze, Gaverni et Géricault notamment.

\section{RÉSUMÉS}

Partant du constat que, malgré la place croissante qu'ils occupent dans nos vies quotidiennes, les systèmes signalétiques n'ont pas fait l'objet jusqu'à maintenant d'une réflexion véritablement approfondie, cet article propose de poser les jalons d'une analyse pluridisciplinaire de ces dispositifs. Leur nature, les logiques d'acteurs qui sous-tendent leur mise en œuvre, leurs fonctions d'orientation mais aussi d'information et de communication ainsi que leurs limites esthétiques et idéologiques sont ainsi évaluées, par le biais d'exemples de réalisations tant urbaines que de musées. Une attention particulière est portée sur la relation qu'entretiennent ces systèmes avec le temps, celui des parcours et celui de l'espace représenté.

This article starts with the observation that, despite the growing role that they play in our lives, information sign systems have not yet been the object of any in-depth thinking. The article proposes to outline a pluridisciplinary analysis of these devices. Their nature, the logics behind their installation, their function in directing but also in informing and communicating as well as their aesthetic and ideological limits are all evaluated by looking at examples in urban environments and museums. Particular attention is paid to the way these systems relate to time, the route taken and the space represented.

\section{AUTEUR}

\section{DOMINIQUE PAGES}

Née le 18 décembre 1958 à Paris, l'auteur est maître de conférences au CELSA, 71e section, responsable pédagogique du magistère de communication. Elle a un doctorat en littérature comparée (1985), Université Paris IV Sorbonne et est une ancienne étudiante du CELSA. 